phys. stat. sol. (b) 139, 399 (1987)

Subject classification: 64.10

Institut für Theoretische und Angewandte Physik der Universität Stuttgart, and. Institut für Physik, Max-Planck-Institut für Metallforschung, Stuttgart

\title{
On the Determination of Interaction Parameters from Correlations in Binary Alloys
}

\author{
By \\ W. MAYSENHÖLDER $\left.{ }^{2}\right)$
}

\begin{abstract}
The determination of interaction parameters from correlation functions in binary alloys is discussed by means of high-temperature expansions for the correlations. The inherent nonlinearity of this "inverse problem" of statistical mechanics leading to non-unique solutions is emphasized. The formulation includes pair and triplet interactions. Some results of a specific "inverse Monte Carlo calculation" are criticized. The paper provides a collection of formulae, especially for probabilities and correlations, which should prove useful for clarifying the subject.
\end{abstract}

Die Bestimmung von Wechselwirkungsparametern aus Korrelationsfunktionen in binären Legierungen wird mittels Hochtemperaturentwicklungen für die Korrelationen diskutiert. Die inhärente Nichtlinearität dieses "inversen Problems" der statistischen Mechanik, das zu nicht-eindeutigen Lösungen führt, wird betont. Die Formulierung schließt Paar- und Triplett-Wechselwirkungen ein. Einige Ergebnisse einer spezifischen ,,inversen Monte-Carlo-Berechnung “ werden kritisiert. Die Arbeit liefert eine Sammlung von Formeln, speziell für Wahrscheinlichkeiten und Korrelationen, die sich als nützlich für die Klärung des Problems erweisen sollten.

\section{Introduction}

In statistical physics theoreticians usually ask the following question: Given the interactions between the particles of the system under consideration, what are the properties of the system as a function of temperature or other external variables? Much less attention is paid to the "inverse problem", the determination of the interactions between the particles from the properties of the system. This may be due to the fundamental difference between these two tasks: For the first one the formal solution is known and unique (we confine ourselves to thermal equilibrium), whereas for the second there is neither a general formal solution nor a guarantee for uniqueness. Nevertheless, the inverse problem deserves attention because the method for the determination of interactions from first principles, quantum mechanics, faces enormous difficulties when accurate and reliable results are required for many-particle systems like liquids and solids (for an example see [1]).

The common way of tackling this inverse problem is as follows: Assume a hopefully adequate structure for the Hamiltonian with some adjustable parameters, solve the direct rather than the inverse problem, and adjust the parameters to make the results agree with experiment. If the last step cannot be realized, the whole procedure has to be repeated with a modified trial Hamiltonian until agreement with experiment can be achieved. If the solution for the parameters is not unique, the set of exper-

1) Pfaffenwaldring 57, D-7000 Stuttgart 80, FRG.

2) Now at Fraunhofer-Institut für Bauphysik, Nobelstr. 12, D-7000 Stuttgart 80, FRG. 
imental values should be augmented until all ambiguity is removed. However, even if all difficulties of the described procedure could be overcome, all we really know is, strictly speaking, that the trial Hamiltonian with certain values for its parameters is compatible with the set of experimental values which has been used for adjusting the parameters. Any extrapolation to other experimental quantities is without rigorous justification, since a different trial Hamiltonian may exist which is also compatible with the original set of experimental values, but leads to different results for other experimental quantities.

Confidence in a particular trial Hamiltonian, i.e. its reliability for predicting experimental quantities which are not automatically reproduced (its "predictive power"), may come from two sources: from physical intuition (which may be wrong) and from quantum-mechanical calculations (which usually are prohibitively difficult). This situation is unpleasant, but it is certainly better to be aware of it than to ignore it.

The present paper serves to illustrate the preceding general considerations in the field of binary alloys at temperatures above the order-disorder transition. It has been stimulated by recent calculations of interactions from short-range order measurements by means of an "inverse Monte Carlo method" [2 to 4]. We do not explain this method here. We rather aim at a critical discussion of the "inverse problem" on the basis of a collection of important formulae. Particular emphasis is given to threebody interactions, the most frequently considered member of many-body interactions. Most equations have been published elsewhere, but some of them are obviously not widely known.

\section{Transformation of the Hamiltonian}

We start from the Hamiltonian for a rigid perfect lattice consisting of $A$ and $B$ atoms, which interact via two- and three-body forces,

$$
H=\frac{1}{2 !} \sum_{\substack{i j \\ \lambda, \mu}} V_{i j}^{\lambda \mu} \sigma_{i}^{\lambda} \sigma_{j}^{\mu}+\frac{1}{3 !} \sum_{\substack{i j k \\ \lambda \mu \nu}} V_{i j k}^{\lambda \mu \nu} \sigma_{i}^{\lambda} \sigma_{j}^{\mu} \sigma_{k}^{v} .
$$

The interaction parameters $V_{i j}^{\lambda \mu}$ and $V_{i j k}^{\lambda \mu \nu}$ are understood to be zero whenever two or three of the site labels $i, j, k$ coincide; thus the summations in (1) need not be restricted. The site labels run from 1 to the total number of atoms $N$. The occupation number $\sigma_{i}^{\lambda}$ is equal to one if site $i$ is occupied by a $\lambda$ atom $(\lambda, \mu, v=\mathrm{A}$ or $\mathrm{B})$ and zero otherwise. As a consequence, $\sigma_{i}^{A}+\sigma_{i}^{B}=1$. It has proved convenient to introduce the transformation

$$
\sigma_{i}=2\left(\sigma_{i}^{\mathrm{A}}-m_{\mathrm{A}}\right)=2\left(m_{\mathrm{B}}-\sigma_{i}^{\mathrm{B}}\right)
$$

with the concentrations of $\mathrm{A}$ and $\mathrm{B}$ atoms, $m_{\mathrm{A}}+m_{\mathrm{B}}=1$. Substitution of (2) in (1) leads to

$$
\begin{aligned}
& H=H_{0}+\frac{1}{4} \sum_{i j} V_{i j} \sigma_{i} \sigma_{j}+\frac{1}{6} \sum_{i j k} V_{i j k} \sigma_{i} \sigma_{j} \sigma_{k} \\
& H_{0}=-\frac{1}{2} \sum_{\substack{i j \\
\lambda \mu}} m_{\lambda} m_{\mu}\left\{V_{i j}^{\lambda, \mu}+\frac{1}{3} \sum_{\substack{k \\
v}} m_{v} V_{i j k}^{\lambda \mu \nu}\right\} \text {, } \\
& V_{i j}=\frac{1}{2}\left\{V_{i j}^{\mathrm{AA}}+\sum_{k}\left(m_{\mathrm{A}} V_{i j k}^{\mathrm{AAA}}+m_{\mathrm{N}} V_{i j k}^{\mathrm{AAB}}\right)+V_{i j}^{\mathrm{BB}}+\sum_{k}\left(m_{\mathrm{A}} V_{i j k}^{\mathrm{BBA}}+m_{\mathrm{B}} V_{i j k}^{\mathrm{BBB}}\right)-\right. \\
& \left.-2 V_{i j}^{\mathrm{AB}}-2 \sum_{k}\left(m_{\mathrm{A}} V_{i j k}^{\mathrm{ABA}}+m_{\mathrm{B}} V_{i j k}^{\mathrm{BAB}}\right)\right\} \\
& \begin{aligned}
V_{i j k}= & \frac{1}{8}\left\{V_{i j k}^{\mathrm{AAA}}-\left(V_{j j k}^{\mathrm{AAB}}+V_{i j k}^{\mathrm{BAA}}+V_{i j k}^{\mathrm{ABA}}\right)+\right. \\
& \left.+\left(V_{i j k}^{\mathrm{BBA}}+V_{i j k}^{\mathrm{ABB}}+V_{i j k}^{\mathrm{BAB}}\right)-V_{i j k}^{\mathrm{BBB}}\right\}
\end{aligned} \\
& \begin{aligned}
V_{i j k}= & \frac{1}{8}\left\{V_{i j k}^{\mathrm{AAA}}-\left(V_{i j k}^{\mathrm{AAB}}+V_{i j k}^{\mathrm{BAA}}+V_{i j k}^{\mathrm{ABA}}\right)+\right. \\
& \left.+\left(V_{i j k}^{\mathrm{BBA}}+V_{i j k}^{\mathrm{ABB}}+V_{i j k}^{\mathrm{BAB}}\right)-V_{i j k}^{\mathrm{BBB}}\right\}
\end{aligned} \\
& \sum_{i} V_{i j k}^{\mathrm{BAA}}=\sum_{k} V_{i j k}^{\mathrm{ABA}} \text { and } \sum_{k} V_{i j k}^{\mathrm{ABB}}=\sum_{k} V_{i j k}^{\mathrm{BAB}} \text { have been used in the equation for } V_{i j}
\end{aligned}
$$


With the prefactor $1 / 6$ in the last term of (3) we follow the definition of Shirley and Wilkins [5] for the $V_{i j k}$. Terms linear in $\sigma_{i}$ vanish because $\sum_{i=1}^{N} \sigma_{i}=0$. For $m_{\mathrm{A}}=m_{\mathrm{B}}=$ $=1 / 2$ we obtain $\sigma_{i}= \pm 1$, and if $V_{i j k}=0$ the Hamiltonian (3) is equivalent to that of an Ising model without external magnetic field.

The equation for $V_{i j}$ can be written in a less symmetric form,

$$
\begin{aligned}
V_{i j}=\frac{1}{2} & \left(V_{i j}^{\mathrm{AA}}+V_{i j}^{\mathrm{BB}}-2 V_{i j}^{\mathrm{AB}}\right)+ \\
& +\frac{1}{2} \sum_{k}\left(V_{i j k}^{\mathrm{AAB}}+V_{i j k}^{\mathrm{BBB}}-2 V_{i j k}^{\mathrm{ABB}}\right)+4 m_{\mathrm{A}} \sum_{k} V_{i j k} .
\end{aligned}
$$

If all the $V$ 's on the right-hand side of (4) were independent of concentration, then a dependence of $V_{i j}$ on $m_{\mathrm{A}}$ would indicate the presence of three-body interactions.

The Hamiltonian (1) could be simplified considerably by the transformation (2). We note that only combinations of the original interaction parameters enter the transformed Hamiltonian; the original ones cannot be retrieved unequivocally from the $V_{i j}$ and $V_{i j k}$.

\section{Correlations and Probabilities}

The short-range order in a disordered alloy can be characterized by correlation functions which are defined as thermodynamical averages of products of $\sigma_{i}$ 's, e.g. the pair correlation function

where

$$
\left\langle\sigma_{i} \sigma_{j}\right\rangle=\frac{\sum \sigma_{i} \sigma_{j} \varrho}{\sum \varrho},
$$

$$
\varrho=\exp \left\{-\beta H\left(\left\{\sigma_{i}\right\}\right)+\Lambda \sum_{i} \sigma_{i}\right\}
$$

is the grand canonical density operator $\left(\beta=1 / k_{\mathrm{B}} T\right)$. The "chemical potential" $A$ must be chosen such that $\left\langle\sigma_{i}\right\rangle=0$. The summations in (5) run over all $N$ ! configurations $\left\{\sigma_{i}\right\}$ of the system.

We also introduce pair and triplet probabilities: $w_{i j}^{\mu \nu}$ is the probability of finding a $\mu$ atom on site $i$ and a $\nu$ atom on site $j \neq i$. Summing over $\mu$ and $\nu$ gives

$$
w_{i j}^{\mathrm{AA}}+w_{i j}^{\mathrm{AB}}+w_{i j}^{\mathrm{BA}}+w_{i j}^{\mathrm{BB}}=1 .
$$

The conditional probabilities $p_{i}^{\mu}{ }_{j}^{v}$ (with the condition behind the vertical bar) connect the pair probabilities with the "one-atom probabilities", the concentrations $m_{\mathbf{A}}$ and $m_{\mathbf{B}}$

$$
w_{i j}^{\mu \nu}=\left.m_{\nu} p_{j}^{\mu}\right|_{i} ^{\nu}=\left.m_{\mu} p_{j}^{\nu}\right|_{i} ^{\mu} .
$$

Because of the three equations

$$
\left.p_{i}^{\mathrm{A}}\right|_{j} ^{v}+\left.p_{i}^{\mathrm{B}}\right|_{j} ^{\nu}=1, \quad w_{i j}^{\mathrm{AB}}=w_{i j}^{\mathrm{BA}}
$$

all pair probabilities referring to sites $i$ and $j$ can be expressed by a single parameter

$$
\alpha_{i j}=1-\frac{\left.p_{i}^{\mathbf{A}}\right|_{j} ^{\mathbf{B}}}{m_{\mathbf{A}}},
$$


which is called Warren-Cowley short-range order parameter [6]. We obtain

$$
\begin{aligned}
& w_{i j}^{\mathrm{A}}=m_{\mathrm{A}}^{2}+m_{\mathrm{A}} m_{\mathrm{B}} \alpha_{i j}, \\
& w_{i j}^{\mathrm{BB}}=m_{\mathrm{B}}^{2}+m_{\mathrm{A}} m_{\mathrm{B}} \alpha_{i j}, \\
& w_{i j}^{\mathrm{AB}}=w_{i j}^{\mathrm{BA}}=m_{\mathrm{A}} m_{\mathrm{B}}\left(\mathbf{1}-\alpha_{i j}\right) .
\end{aligned}
$$

For complete disorder $\alpha_{i j}=0$, for complete segregation $\alpha_{i j}=1$. Since all probabilities are restricted to values between zero and one, the Warren-Cowley parameter is restricted, too,

$$
-1 \leqq-\frac{m_{\mathrm{A}}}{m_{\mathrm{B}}} \leqq \alpha_{i j} \leqq 1
$$

(Without loss of generality we assume $m_{\mathrm{A}} \leqq m_{\mathrm{B}} \neq 0$.) Finally, combination of (10) and (11) yields

$$
\begin{aligned}
& 0 \leqq w_{i j}^{\mathrm{A}} \leqq m_{\mathrm{A}} \\
& m_{\mathrm{B}}-m_{\mathrm{A}} \leqq w_{i j}^{\mathrm{BB}} \leqq m_{\mathrm{B}}, \\
& 0 \leqq w_{i j}^{\mathrm{AB}}=w_{i j}^{\mathrm{BA}} \leqq m_{\mathrm{A}},
\end{aligned}
$$

i.e. the pair probabilities are restricted by the concentrations.

The relationship between probabilities and correlations is easily derived if we observe that $(i \neq j)$

$$
\left\langle\sigma_{i}^{\mu}\right\rangle=m_{\mu}, \quad\left\langle\sigma_{i}^{\mu} \sigma_{j}^{\nu}\right\rangle=w_{i j}^{\mu \nu} .
$$

Using (2) we obtain for the pair correlations $(i \neq j)$

$$
\left\langle\sigma_{i} \sigma_{j}\right\rangle=4\left(w_{i j}^{\mathrm{AA}}-m_{\mathrm{A}}^{2}\right)=4 m_{\mathrm{A}} m_{\mathrm{B}} \alpha_{i j} .
$$

Accordingly, we calculate for the triplet correlations $(i \neq j \neq k \neq i)$

$$
\begin{aligned}
\left\langle\sigma_{i} \sigma_{j} \sigma_{k}\right\rangle & =8\left\{w_{i j k}^{\mathrm{AAA}}-m_{\mathrm{A}}\left(w_{i j}^{\mathrm{AA}}+w_{j k}^{\mathrm{AA}}+w_{k i}^{\mathrm{AA}}\right)+2 m_{\mathrm{A}}^{3}\right\} \\
& =8\left\{w_{i j k}^{\mathrm{AAA}}-m_{\mathrm{A}}^{3}-m_{\mathrm{A}}^{2} m_{\mathrm{B}}\left(\alpha_{i j}+\alpha_{j k}+\alpha_{k i}\right)\right\} .
\end{aligned}
$$

The eight triplet probabilities $w_{i j k}^{\lambda \mu \nu}$ can be written as functions of the concentrations, the Warren-Cowley parameters, and one of the triplet probabilities, say $w_{i j k}^{\mathrm{AAA}}$ (there is no Warren-Cowley parameter for triplets),

$$
\begin{aligned}
& w_{i j k}^{\mathrm{AAA}}=w_{i j k}^{\mathrm{AAA}}, \\
& w_{i j k}^{\mathrm{AAB}}=m_{\mathrm{A}}^{2}+m_{\mathrm{A}} m_{\mathrm{B}} \alpha_{i j}-w_{i j k}^{\mathrm{AAA}}, \\
& w_{i j k}^{\mathrm{BAA}}=m_{\mathrm{A}}^{2}+m_{\mathrm{A}} m_{\mathrm{B}} \alpha_{j k}-w_{i j k}^{\mathrm{AAA}}, \\
& w_{i j k}^{\mathrm{ABA}}=m_{\mathrm{A}}^{2}+m_{\mathrm{A}} m_{\mathrm{B}} \alpha_{k i}-w_{i j k}^{\mathrm{AAA}}, \\
& w_{i j k}^{\mathrm{BAB}}=-m_{\mathrm{A}}^{2}+m_{\mathrm{A}} m_{\mathrm{B}}\left(1-\alpha_{i j}-\alpha_{j k}\right)+w_{i j k}^{\mathrm{AAA}}, \\
& w_{i j k}^{\mathrm{ABB}}=-m_{\mathrm{A}}^{2}+m_{\mathrm{A}} m_{\mathrm{B}}\left(1-\alpha_{k i}-\alpha_{i j}\right)+w_{i j k}^{\mathrm{AAA}}, \\
& w_{i j k}^{\mathrm{BBA}}=-m_{\mathrm{A}}^{2}+m_{\mathrm{A}} m_{\mathrm{B}}\left(1-\alpha_{j k}-\alpha_{k i}\right)+w_{i j k}^{\mathrm{AAA}}, \\
& w_{i j k}^{\mathrm{BBB}}=1+m_{\mathrm{A}} m_{\mathrm{B}}\left(\alpha_{i j}+\alpha_{j k}+\alpha_{k i}-3\right)-w_{i j k}^{\mathrm{AAA}} .
\end{aligned}
$$

The corresponding set of equations with $\left\langle\sigma_{i} \sigma_{j} \sigma_{k}\right\rangle$ instead of $w_{i j k}^{\mathrm{AAA}}$ on the right-hand side has been given by Clapp [7]. 
If all triplet probabilities are known, we can calculate the pair probabilities, e.g.

$$
w_{i j}^{\mathrm{AB}}=w_{i j k}^{\mathrm{ABA}}+w_{i j k}^{\mathrm{ABB}}=m_{\mathrm{A}} m_{\mathrm{B}}\left(1-\alpha_{i j}\right),
$$

and from the pair probabilities the concentrations, e.g.

$$
m_{\mathrm{A}}=w_{i j}^{\mathrm{A}}+w_{i j}^{\mathrm{AB}}=m_{\mathrm{A}}^{2}+m_{\mathrm{A}} m_{\mathrm{B}}=m_{\mathrm{A}} \text {. }
$$

On the other hand, if we start from the concentrations and want to specify pair correlations, we have to observe restriction (11); similarly, we are not completely free in specifying the triplet probabilities. The 16 inequalities

$$
0 \leqq w_{i j k}^{\lambda \mu v} \leqq 1
$$

lead to 16 inequalities for $w_{i j k}^{\mathrm{AA}}$ which can be summarized symbolically as

$$
\max \left\{f_{n}\left(m_{\mathrm{A}}, \alpha_{i j}, \alpha_{j k}, \alpha_{k i}\right)\right\} \leqq w_{i j k}^{\mathrm{AAA}} \leqq \min \left\{g_{n}\left(m_{\mathrm{A}}, \alpha_{i j}, \alpha_{j k}, \alpha_{k i}\right)\right\}
$$

with the functions $f_{n}$ and $g_{n}$ depending on the concentrations and the pair correlations $(n=0 \ldots 7)$. Condition (18) can be satisfied only if the maximum of the left-hand side is not greater than the minimum of the right-hand side. This means that for a given concentration $m_{\mathrm{A}}$ the pair correlations $\alpha_{i j}, \alpha_{j k}, \alpha_{k i}$ might be restricted by the mere existence of triplet $i j k$ !

Since the case of a general triplet $i j k$ is complex, we illustrate these restrictions for a triplet forming an equilateral triangle. Equations (16) now simplify to

$$
\begin{aligned}
& w^{\mathrm{AAA}}=w^{\mathrm{AAA}}, \\
& w^{\mathrm{AAB}}=m_{\mathrm{A}}^{2}+m_{\mathrm{A}} m_{\mathrm{B}} \alpha-w^{\mathrm{AAA}}, \\
& w^{\mathrm{BBA}}=-m_{\mathrm{A}}^{2}+m_{\mathrm{A}} m_{\mathrm{B}}(\mathbf{1}-2 \alpha)+w^{\mathrm{AAA}}, \\
& w^{\mathrm{BBB}}=1+3 m_{\mathrm{A}} m_{\mathrm{B}}(\alpha-1)-w^{\mathrm{AAA}},
\end{aligned}
$$

with the normalization

$$
w^{\mathrm{AAA}}+3 w^{\mathrm{AAB}}+3 w^{\mathrm{BBA}}+w^{\mathrm{BBB}}=1 .
$$

(We have dropped the site labels for convenience.) Condition (18) becomes

with

$$
\max \left\{0, f_{1}, f_{2}, f_{3}\right\} \leqq w^{\mathrm{AAA}} \leqq \min \left\{1, g_{1}, g_{2}, g_{3}\right\}
$$

$$
\begin{array}{ll}
f_{1}=m_{\mathrm{A}}^{2}+m_{\mathrm{A}} m_{\mathrm{B}} \alpha-\frac{1}{3}, & g_{1}=f_{1}+\frac{1}{3}, \\
f_{2}=m_{\mathrm{A}}^{2}-m_{\mathrm{A}} m_{\mathrm{B}}(1-2 \alpha), & g_{2}=f_{2}+\frac{1}{3}, \\
f_{3}=3 m_{\mathrm{A}} m_{\mathrm{B}}(\alpha-1), & g_{3}=f_{3}+1 .
\end{array}
$$

Evaluation of (20) leads to three regions (I to III) in a $m_{\mathrm{A}}-\alpha$-diagram with different bounds for $w^{\mathrm{AAA}}$ and a "forbidden" region (IV) with combinations of $m_{\mathrm{A}}$ - and $\alpha$-values which can never occur. Fig. 1 a shows these regions separated by the following curves:

$$
\begin{aligned}
\text { I-II: } \quad \alpha & =1-\frac{1}{2 m_{\mathrm{B}}}, \\
\text { II-III : } \quad \alpha & =1-\frac{1}{2 m_{\mathrm{A}}}, \\
\text { II-IV : } \quad \alpha & =-\frac{m_{\mathrm{A}}}{m_{\mathrm{B}}}, \\
\text { III-IV : } \quad \alpha & =1-\frac{1}{3 m_{\mathrm{A}} m_{\mathrm{B}}} .
\end{aligned}
$$



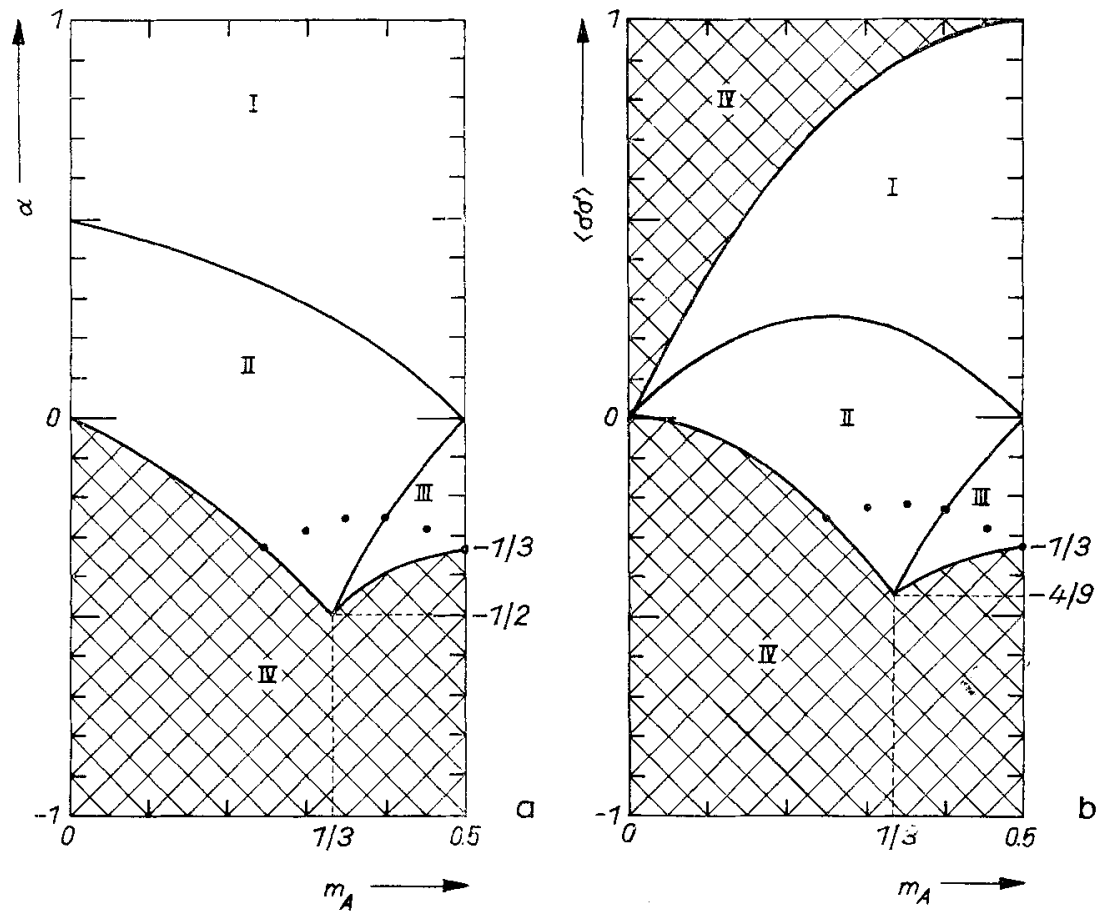

Fig. 1. Regions I-III of possible combinations of concentration $m_{A}$ and a) Warren-Cowley parameter $\alpha$ or b) pair correlation $\langle\sigma \sigma\rangle$ with different bounds on the triplet probability $u^{A A A}$. The crosshatched region IV is "forbidden". For dotted lines and other details see text

The last curve (24) can immediately be obtained from (19)| if we demand $w^{\text {AAA }}+$ $+w^{\mathrm{BBB}} \geqq 0$. For $\alpha$-values below this curve "max" in $(20)$ is greater than "min": The mere existence of triplets excludes these $\alpha$-values. As an aside we note that regions II and III are further reduced by the existence of quadruplets which form a regular tetrahedron (e.g. in f.c.c. and h.c.p. structures), namely by the curve

$$
\alpha=1-\frac{2 m_{\mathrm{A}}+1}{6 m_{\mathrm{A}} m_{\mathrm{B}}},
$$

which is the dotted line in Fig. 1 a. The bounds for $w^{\text {AAA }}$ are given by

$$
\begin{aligned}
\text { I: } & f_{2} \leqq w^{\mathrm{AAA}} \leqq g_{1}, \\
\mathrm{II}: & 0 \leqq w^{\mathrm{AAA}} \leqq g_{1}, \\
\mathrm{III}: & 0 \leqq w^{\mathrm{AAA}} \leqq g_{3} .
\end{aligned}
$$

This is illustrated in Fig. 2 a for several values of $m_{\mathrm{A}}\left(w^{\mathrm{AAA}}=m_{\mathrm{A}}\right.$ for $\left.\alpha=1\right)$. By means of (14) and (15) we can easily deduce corresponding relations for $m_{A},\langle\sigma \sigma\rangle$, and $\langle\sigma \sigma \sigma\rangle$ (see Fig. $1 \mathrm{~b}$ and $2 \mathrm{~b}$ ). When tetrahedral quadruplets exist, there will be additional restrictions in Fig. 2.

The effect of higher-order clusters of atoms on $\langle\sigma \sigma\rangle$ and $\langle\sigma \sigma \sigma\rangle$ has presumably not been investigated yet. (Some bounds for triplet probabilities have already been calculated by Clapp [8] and by Gragg et al. [9].) 

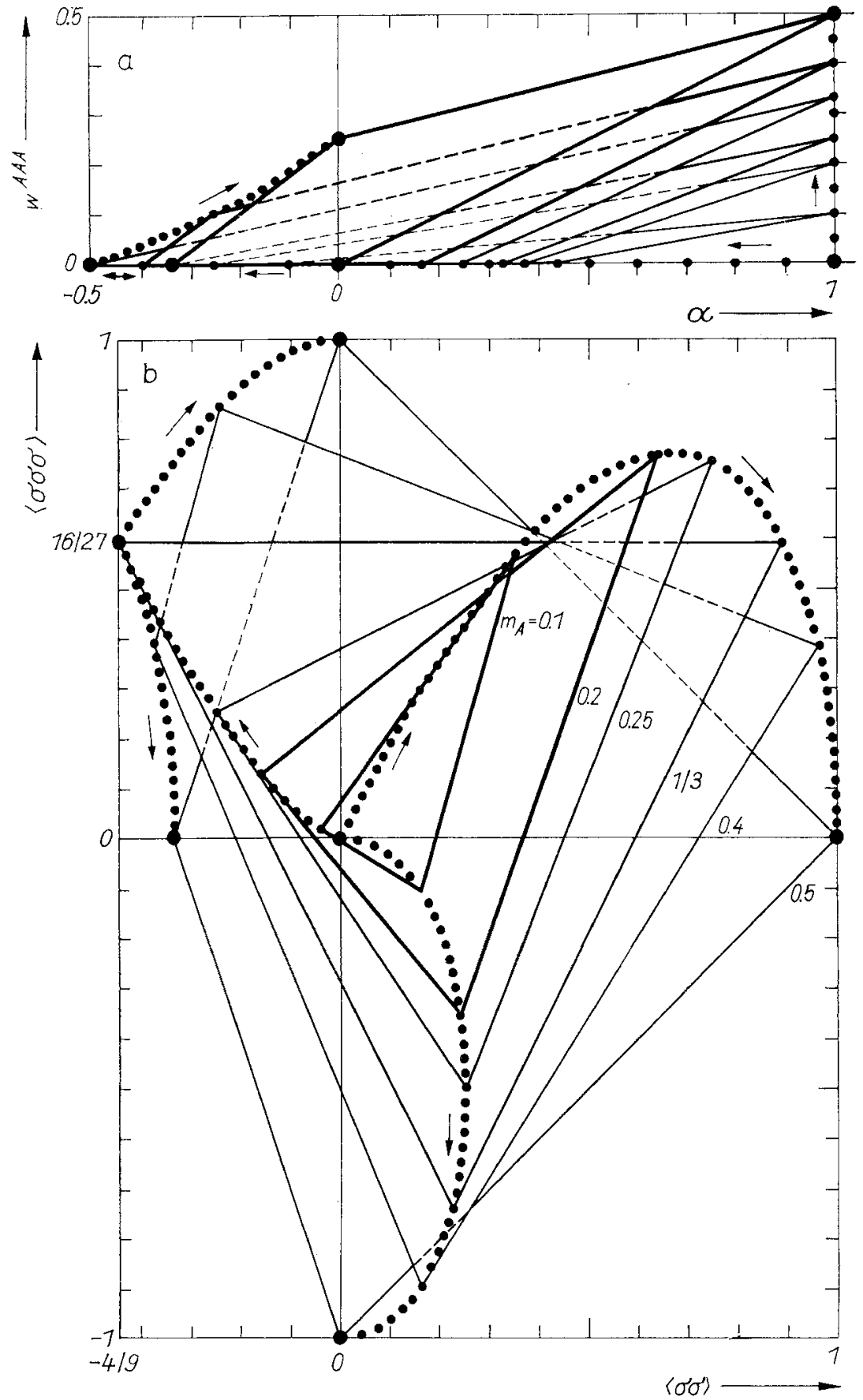

Fig. 2. Possible range of a) $w^{\mathrm{AAA}}$ as a function of $\alpha$ and of b) the triplet correlation $\langle\sigma \sigma \sigma\rangle$ as a function of $\langle\sigma \sigma\rangle$ for various values of $m_{\mathbf{A}}\left(m_{\mathbf{A}}=w^{\mathrm{AAA}}\right.$ for $\left.\alpha=1\right)$. The regions outside the triangles and quadrangles are "forbidden". The corners of these polygons lie on the dotted lines with arrows marking the direction of increasing $m_{\mathrm{A}}$. (Bold and dashed lines are to suggest a three-dimensional appearance.) 


\section{High-Temperature Expansions}

The Hamiltonian (3) with interaction parameters $V_{i j}$ and $V_{i j k}$ uniquely determines the correlation functions by means of relations from statistical mechanics such as (5). As in the case of the Ising model analytical results for the correlations are known only for special cases, especially for high temperatures. Except for $m_{\mathrm{A}}=m_{\mathrm{B}}$, where the results of the Ising model without magnetic field can be transferred, evaluation of the leading terms of the high-temperature expansion requires even more theoretical effort than the standard procedure for the Ising model including magnetic field (see, e.g., [10]). This is mainly due to the fact that the "chemical potential" $A$, which in the grand canonical ensmble is responsible for the desired concentrations $m_{\mathrm{A}}$ and $m_{\mathfrak{B}}$, is not weighted by $\beta$ in the density operator $\varrho$ like a magnetic field. Thus high-temperature expansions for binary alloys are more involved.

An elegant method of solution has been worked out by Clapp and Moss [11]. They arrive at the implicit equations

$$
\alpha_{i j} \approx-2 m_{\mathrm{A}} m_{\mathrm{B}} \beta \sum_{f} V_{i f} \alpha_{f j} \quad(i \neq j)
$$

and solve them for $V_{i j}$ or $\alpha_{i j}$ by Fourier transformation. It should be noted that the high-temperature expansion used in [11] has temperature-dependent coefficients; therefore terms of different order may contribute to one of the (temperature-independent) coefficients of the expansion in powers of $\beta$. To first order we obtain from (27)

$$
\left\langle\sigma_{i} \sigma_{j}\right\rangle=4 m_{\mathrm{A}} m_{\mathrm{B}} \alpha_{i j} \approx-8 m_{\mathrm{A}}^{2} m_{\mathrm{B}}^{2} \beta V_{i j} \quad(i \neq j)
$$

because $\alpha_{i j}=1$. The method of Clapp and Moss can be generalized to three-body interactions (the "chemical potential" $A$ then depends on the $V_{i j k}$, however!),

$$
\left\langle\sigma_{i} \sigma_{j} \sigma_{k}\right\rangle \approx-64 m_{\mathrm{A}}^{3} m_{\mathrm{j} ;}^{3} \beta V_{i j k} \quad(i \neq j \neq k \neq i) .
$$

Clapp [7] has shown that in the special case $m_{\mathrm{A}}=m_{\mathrm{B}}$ and $V_{i j k}=0$ the triplet correlations and also all other odd-order correlations vanish.

Extensive work on high-temperature expansions including three-body interactions has been performed by Taggart and Tahir-Keli [12] and by Shirley and Wilkins [5]. Unfortunately, the results of Taggart and Tahir-Keli are not very transparent (presumably due to an unfavourable transformation) and practically useless.

Shirley and Wilkins give expressions (which look relatively manageable) for pair, triplet, and quadruplet correlations up to second order in $\beta$ ((12) to (14) of [5]). For the purpose of our discussion it suffices to recall the expression for pair correlations,

$$
\begin{aligned}
& \left\langle\sigma_{i} \sigma_{j}\right\rangle \approx-8 m_{\mathrm{A}}^{2} m_{\mathrm{B}}^{2} V_{i j} \beta+\left[16 m_{\mathrm{A}}^{3} m_{\mathrm{B}}^{3} \sum_{f} V_{i f} V_{f j}+\right. \\
& +64 m_{\mathrm{A}}^{3} m_{\mathrm{B}}^{3}\left(m_{\mathrm{B}}-m_{\mathrm{A}}\right) \sum_{j}\left(V_{i f}+V_{j f}\right) V_{i j f}+8 m_{\mathrm{A}}^{2} m_{\mathrm{B}}^{2}\left(m_{\mathrm{B}} m_{\mathrm{A}}\right)^{2} V_{i j}^{2}+ \\
& \left.+128 m_{\mathrm{A}}^{4} m_{\mathrm{B}}^{4} \sum_{f g} V_{i f g} V_{j f g}+128 m_{\mathrm{A}}^{3} m_{\mathrm{B}}^{3}\left(m_{\mathrm{B}}-m_{\mathrm{A}}\right)^{2} \sum_{f} V_{i f j}^{2}\right] \beta^{2} \quad(i \neq j) .
\end{aligned}
$$

The important feature of this equation is the occurrence of products of interaction parameters. Hence, a unique determination of interaction parameters from correlation functions cannot be expected in general.

\section{Discussion of the Inverse Problem}

The inverse problem, i.e. determination of interaction parameters from correlations, is solved simply and uniquely if the linear equations (28), (29), or (27) are used. Since 
triplet correlations have not been measured yet, one might think of a simultaneous determination of pair and triplet interactions from the nonlinear equations (30). However, it should be obvious from (30) that this cannot be done in a unique manner, at least if the pair correlations are known only for one concentration and one temperature. But even if the pair correlations are known for various concentrations and temperatures, additional difficulties are introduced by the concentration and temperature dependence of the interaction parameters: These depend on the concentrationand temperature-dependent lattice parameter and also on the concentration-dependent Fermi surface, which affects period and phase of the Friedel oscillations.

For the case $m_{\mathrm{A}}=m_{\mathrm{B}}=1 / 2(30)$ simplifies considerably,

$$
\left\langle\sigma_{i} \sigma_{j}\right\rangle \approx-\frac{1}{2} V_{i j} \beta+\left[\frac{1}{4} \sum_{f} V_{i f} V_{f j}+\frac{1}{2} \sum_{f g} V_{i f g} V_{j f g}\right] \beta^{2} \quad(i \neq j) .
$$

If the V's were independent of temperature, $(31)$ could be used for estimates of triplet interactions. Expansions of $V_{i j}$ and $V_{i j k}$ around some reference temperature, e.g.

$$
V_{i j} \approx V_{i j}\left(\beta_{\mathrm{r}}\right)+t_{i j}\left(\frac{1}{\beta}-\frac{1}{\beta_{\mathrm{r}}}\right)
$$

could be inserted in (31) as a refinement but at the cost of additional unknowns $\left(t_{i j}, t_{i j k}\right)$.

Schweika and Haubold [4] determined $V_{i j}$ from measured values $\alpha_{i j}$ for the binary alloy $\mathrm{Ni}_{\mathbf{0} 89} \mathrm{Cr}_{\mathbf{0 . 1 1}}$ both by the solution of the Clapp and Moss equation (27) and by the "inverse Monte Carlo method" suggested by Gerold and Kern [2,3]. The results obtained by the two methods are in close agreement with each other except for the nearest-neighbour pair interaction, for which the condition $\beta V_{i j} \leqslant 1$ necessary for the validity of $(27)$ is no longer satisfied $\left(\beta V_{i j} \approx 0.36\right)$. Both the $V_{i j}$ and the $\alpha_{i j}$ nicely show Friedel oscillations.

By means of the "inverse Monte Carlo method" Schweika and Haubold also tried to determine some three-body interactions. For instance, they obtained $2.2 \pm$ $\pm 0.5 \mathrm{meV}$ for the case of the nearest-neighbour equilateral triangle leading according to (29) - to a negative triplet correlation. However, if we solve (15) for the triplet probability $w^{\text {AAA }}$,

$$
w^{\mathrm{AAA}}=m_{\mathrm{A}}^{3}+3 m_{\mathrm{A}}^{2} m_{\mathrm{B}} \alpha+\frac{1}{8}\langle\sigma \sigma \sigma\rangle,
$$

and insert the experimental nearest-neighbour pair correlations $\alpha=-0.0543$ and $m_{\mathrm{A}}=0.11$, the resulting triplet probability $w^{\mathrm{AAA}}$ comes out negative! We have to conclude that the Monte Carlo result cannot be meaningful, because (33) follows rigorously from the definition (2) and relations of type (13) between correlations and probabilities. (The fact that the triplet correlations are restricted by the pair correlations does not help at all in the determination of triplet interactions.) This illustrates the usefulness of (15) as a check on the compatibility of correlations. The conditions $0 \leqq w_{i j k}^{A A A} \leqq 1$ or $(20)$ and its generalizations together with (15) can be used to determine the possible range of triplet correlations. In our example we conclude that $\langle\sigma \sigma \sigma\rangle$ has to be positive, hence - if (29) applies -- the corresponding triplet interaction is negative. This kind of reasoning can render more detailed calculations on the basis of (30) or with Monte Carlo methods simpler and less ambiguous.

\section{Conclusions}

We have briefly touched the problem of determining interaction parameters from correlation functions. Except where the lowest-order high-temperature expansions $27^{*}$ 
(28), (29) are applicable the problem suffers from the inherent nonlinearity of the equations, which may have more than one solution. For this reason such solutions should be used with some caution in subsequent calculations for the prediction of phase transitions or other properties of the alloy. (Limitations of the pair-interaction model for ordered alloys are discussed for instance by Clapp [13].) In order to obtain reliable three-body interactions, measurements of triplet correlations appear almost unavoidable. Whenever possible, it should be checked that all occurring probabilities come to lie between zero and one. At first sight Monte Carlo methods seem to be able to include many-body interactions without principal difficulties. However, the fundamental nonlinearity and ambiguity of the inverse problem still remain and can be resolved only in favourable circumstances.

\section{Acknowledgements}

The author is grateful for stimulating and helpful discussions with Prof. A. Seeger, Dr. L. Longa. Dr. L. Schimmele, Dr. M. Fähnle, Dr. W. Schweika, and Dr. H.-G. Haubold. Thanks also to C. Maysenhölder for drawing the figures.

\section{References}

[1] A. Bibter and F. Gautier, Z. Phys. B 57, 335 (1984).

[2] V. Gerold and J. Kern, Acta cryst. A40, C432 (1984).

[3] V. GEROLd and J. KERN, in: Atomie Transport and Defects in Metals by Neutron Scattering, Ed. C. Jayot, W. Petry, D. Richter, and T. Springer, Springer-Verlag, Berlin 1986 (p. 17).

[4] W. Schweika and H.-G. HaUbol.D, in: Atomic Transport and Defects in Metals by Neutron Scattering, Ed. C. JAnot, W. Petry, D. Richter, and T. Springer, Springer-Verlag, Berlin 1986 (p. 22).

[5] C. G. Shtrley and S. Wilkins, Phys. Rev. B 6, 1252 (1972).

[6] B. E. WARREN, X-Ray Diffraction, Addison-Wesley, Reading (Mass.) 1969. J. M. Cowley, Phys. Rev. 77, 669 (1950).

[7] P. C. Clapp, Phys. Rev. 164, 1018 (1967).

[8] P. C. Clapp, in: Critical Phenomena in Alloys, Nagnets and Superconductors, Ed. R. E. MrLls, E. Ascher, and R. I. Jaffee, MeGraw-Hill Publ. Co., New York 1971 (p. 299).

[9] J. E. GragG, Jr., P. Bardhan, and J. B. Cohen, in: Critical Phenomena in Alloys, Magnets and Superconductors, Ed. R. E. Mitus, E. Ascher, and R. I. JAffee, MeGraw-Hill Publ. Co., New York 1971 (p. 309).

[10] H. E. Stantey, Introduction to Phase Transitions and Critical Phenomena, Clarendon Press, Oxford 1971.

[1.1] P. C. ClapP and S. C. Moss, Phys. Rev. 142, 418 (1966).

[12] G. B. Taggart and R. A. Tahir-Keli, Progr. theor. Phys. (Kyoto) 46, 1690 (1971).

[13] P. C. Clapp, in: Ordered Alloys, Ed. B. H. Kear, C. T. Sims, N. S. StolofF, and J. H. WestBRook, Claitors, Baton Rouge 1970 (p. 25). 